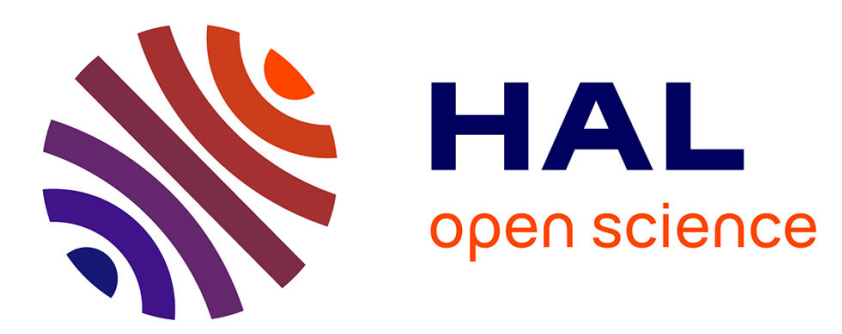

\title{
Design and Evaluation of Methods to Prevent Frame Cancellation in Real-Time Stereoscopic Rendering
}

\author{
Jérôme Ardouin, Anatole Lécuyer, Maud Marchal, Eric Marchand
}

\section{To cite this version:}

Jérôme Ardouin, Anatole Lécuyer, Maud Marchal, Eric Marchand. Design and Evaluation of Methods to Prevent Frame Cancellation in Real-Time Stereoscopic Rendering. IEEE Symposium on 3D User Interfaces, 3DUI 2011, 2011, Singapore, Singapore. pp.95-98. hal-00639649

\section{HAL Id: hal-00639649 \\ https://hal.inria.fr/hal-00639649}

Submitted on 9 Nov 2011

HAL is a multi-disciplinary open access archive for the deposit and dissemination of scientific research documents, whether they are published or not. The documents may come from teaching and research institutions in France or abroad, or from public or private research centers.
L'archive ouverte pluridisciplinaire HAL, est destinée au dépôt et à la diffusion de documents scientifiques de niveau recherche, publiés ou non, émanant des établissements d'enseignement et de recherche français ou étrangers, des laboratoires publics ou privés. 


\section{Design and Evaluation of Methods to Prevent Frame Cancellation in Real-Time Stereoscopic Rendering}

\author{
Jérôme Ardouin * \\ ESIEA/INSA/INRIA
}

\author{
Anatole Lécuyer ${ }^{*}$ \\ INRIA
}

\author{
Maud Marchal \\ INSA/INRIA
}

\author{
Eric Marchand ${ }^{*}$ \\ University of Rennes 1/INRIA
}

\begin{abstract}
Frame cancellation comes from the conflict between two depth cues: stereo disparity and occlusion with the screen border. When this conflict occurs, the user suffers from poor depth perception of the scene. It also leads to uncomfortable viewing and eyestrain due to problems in fusing left and right images.

In this paper we propose a novel method to avoid frame cancellation in real-time stereoscopic rendering. To solve the disparity/frame occlusion conflict, we propose rendering only the part of the viewing volume that is free of conflict by using clipping methods available in standard real-time 3D APIs. This volume is called the "Stereo Compatible Volume" (SCV) and the method is named "Stereo Compatible Volume Clipping" (SCVC).

Black Bands, a proven method initially designed for stereoscopic movies is also implemented to conduct an evaluation. Twenty two people were asked to answer open questions and to score criteria for SCVC, Black Bands and a Control method with no specific treatment. Results show that subjective preference and user's depth perception near screen edge seem improved by SCVC, and that Black Bands did not achieve the performance we expected.

At a time when stereoscopic capable hardware is available from the mass consumer market, the disparity/frame occlusion conflict in stereoscopic rendering will become more noticeable. SCVC could be a solution to recommend. SCVC's simplicity of implementation makes the method able to target a wide range of rendering software from VR application to game engine.
\end{abstract}

Keywords: Stereoscopy, display technology, visualization, stereo compatible volume, clipping.

Index Terms: H.5.2 [Information Interfaces and Presentation]: User Interfaces-user-centered design; I.3.3 [Computer Graphics]: Picture/Image Generation-display algorithms; I.3.7 [Computer Graphics]: Three-Dimensional Graphics and Realism-Virtual Reality

\section{INTRODUCTION}

In stereoscopic real-time rendering, depth perception problems occur when objects are displayed off screen (negative parallaxes) close to the screen border. This effect has been observed since the early works on stereoscopic movies. Valyus has named the phenomenon frame cancellation [1]. The problem comes from the conflict between two depth cues: stereo disparity tells the user that the perceived object is in front of the screen while the occlusion with the screen border indicates that the screen border is in front of the object. When this conflict occurs, the user suffers

"e-mail : \{jardouin, alecuyer, mmarchal, emarchand\}@inria.fr

IEEE Symposium on 3D User Interfaces 2011

19-20 March, Singapore

978-1-4577-0064-4/11/\$26.00 @2011 IEEE poor depth perception of the scene [2]. It also leads to uncomfortable viewing and eyestrain due to problems in fusing left and right images [3].

Figure 1 illustrates the typical configuration of left and right eye viewing volume for a determined display surface. Intersection of the left and right viewing volume is not subject of frame cancellation while the remaining volume represents location of geometry that leads to disparity/frame occlusion conflict (D/FO conflict). Let it be noticed that the positive parallax volume is not pictured since it is not subject to the frame cancellation problem.

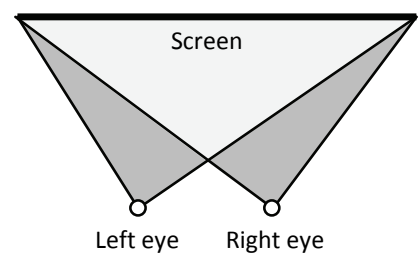

Figure 1. Top view of the left and right camera volumes. Light gray: stereo compatible volume. Dark gray: volume subject to disparity / frame occlusion conflict.

In this paper we propose a novel method to avoid frame cancellation in real-time stereoscopic rendering. This method is based on the observation of the stereoscopic viewing volume shape. To solve the $\mathrm{D} / \mathrm{FO}$ conflict, we propose rendering only the part of the viewing volume that is free of conflict by using clipping methods available in standard real-time 3D APIs. This volume is called "Stereo Compatible Volume" (SCV) and the method is named "Stereo Compatible Volume Clipping" (SCVC).

The paper is organized as follows: section 2 proposes an overview of the existing methods to solve the D/FO conflict, section 3 proposes an analysis of these methods, explaining their limitations and suitability to real-time rendering. Our approach is described in section 4, and then it is evaluated in section 5 .

\section{Related Work}

Although the problem has been assessed using various technical solutions in stereoscopic movies and other offline computed stereoscopic rendering, fewer methods have been developed with real-time rendering specificities in mind.

Methods from movie industry and offline stereoscopic rendering: Some digital content creation products like Autodesk Maya propose to display the intersection of the 2 view volumes (called safe viewing volume). This technique is meant to help the creators in placing objects in situation free of D/FO conflict [4].

The floating window is a common approach. It consists in inserting black bands on the left and right edges of the screen if the displayed content is subject to D/FO conflict [5]. The result is that the perceived content appears to be in positive parallaxes related to a virtual sub window positioned in user space (Figure 2).

Other approaches consist in modifying the disparity of the stereo pair globally or close to the screen edge. This can be seen as a local distortion of the $3 \mathrm{D}$ space, ensuring zero or positive 
parallaxes in areas where frame cancellation occurs. Lipton et al. propose to stretch the stereo pair at the border to insure zero or positive parallaxes while preserving enough negative parallax at the center of the screen so that the viewer experience remains convincing [3].

Modifications of the stereo pair disparity are generalized by Lang et al. [6] who have proposed operators on disparity maps. They are used in different manners to modify disparity of the stereo pair to achieve effects such as emphasizing subject, shifting the scene depth or magnifying the depth range. Given a stereo pair, a disparity map is computed using sparse feature matching. Operators are applied to this map in the way that tonal transformations are applied to bitmap images. Finally the stereo pair is warped to reflect the new disparity map. This is done by employing retargeting techniques [7], preserving the structure of images in both the spatial and temporal domain.

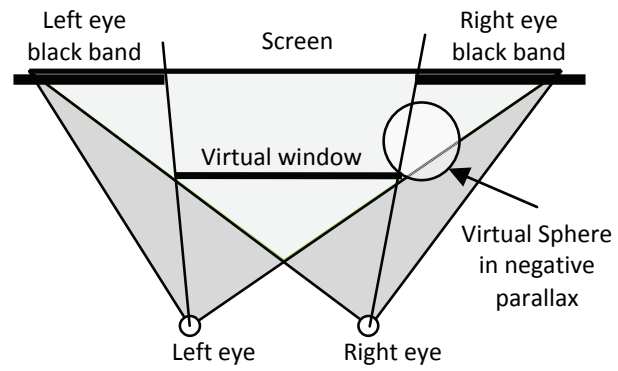

Figure 2. Black bands. The part of the virtual sphere subject to frame cancellation is discarded by restricting the viewing volume with black bands.

Real-time rendering methods: Mulder et al. have proposed a real-time implementation of the Black Bands through the Cadre Viewing approach [8]. An improvement is also proposed with the Tunnel extension. With an overplayed grid, the tunnel can improve depth perception by providing points easy to fuse.

Ware et al. have introduced the concept of cyclopean scale, presented as a rescale of the viewed scene centered on the midpoint between the user's eyes [9]. Magnitude of the scaling is computed to place the virtual environment just behind the screen. This clearly avoids frame cancellation as it shifts the scene to the positive parallax half space. Combined with interactions, controls of scene scale and translation have to be performed dynamically in an intelligent manner. This problem is assessed by Wartell et al. through various derived approaches, by for example analyzing the depth buffer to compute valid parameters [2].

\section{LIMITS OF EXISTING METHODS}

In the category of movie design driven methods, black band insertion is certainly the most adapted to real-time rendering. The main drawback occurs in situations where strong negative parallax objects are subject to $\mathrm{D} / \mathrm{FO}$ conflict. In this kind of configuration, the virtual window can be very small and dramatically reduce the horizontal field of view. The aspect ratio of the view volume is also heavily altered. Another limitation comes from the dependency of the method on scene analysis: to determine the width of black bands we have to test each object against the stereo compatible volume. Also a part of the SCV is discarded along with the problematic portion. Cadre viewing [8] limitations are roughly the same as black bands. The Tunnel extension restricts even more the viewing volume.

The stretch method [3] and disparity map operators [6] are based on the analysis of the disparity of the stereo pair. The disparity map of the stereo pair is usually computed with offline algorithms such as sparse feature matching. A real-time version of these kinds of algorithms could rely on the $\mathrm{Z}$ buffer in conjunction with the left to right eye transformation matrix to generate a disparity map. The remaining limitation is the non constant scale of the perceived scene along screen space that could lead to disturbing effects, especially when used with head tracked display. Disparity map operators also are difficult to use in real-time as retargeting techniques imply specific computation and human guided tuning in some cases. Its need of time forward information to maintain consistency in the image structure over time is another weakness for real-time rendering.

Cyclopean scale and its variations differ radically from the above methods because they have been designed to target realtime rendering and real-time interactions. The application has to comply with scale modification of the displayed scene (with head tracked displays, maintaining 1:1 scale is often an important requirement). The type of geometry to which the method applies is also limited to convex objects so exploration of an open environment is not an option. Cyclopean scale restricts the view volume to positive parallax or very low negative parallax values. Since the negative parallax portion of the viewing volume is an area useful for collocated interactions, this point can be considered as another limitation.

Table 1. Properties and limitations of existing methods for solving disparity/frame occlusion conflict.

\begin{tabular}{|c|c|c|c|c|}
\cline { 2 - 5 } \multicolumn{1}{c|}{} & $\begin{array}{c}\text { Black Bands } \\
{[5][8]}\end{array}$ & $\begin{array}{c}\text { Border } \\
\text { stretching [3] }\end{array}$ & $\begin{array}{c}\text { Disparity } \\
\text { Map [6] }\end{array}$ & $\begin{array}{c}\text { Cyclopean } \\
\text { scale [9][2] }\end{array}$ \\
\hline $\begin{array}{c}\text { Real-time } \\
\text { Compatibility }\end{array}$ & Yes & No & No & Yes \\
\hline $\begin{array}{c}\text { Content analysis } \\
\text { dependency }\end{array}$ & Yes & No & No & Yes \\
\hline $\begin{array}{c}\text { Disparity analysis } \\
\text { dependency }\end{array}$ & No & Yes & Yes & No \\
\hline $\begin{array}{c}\text { Scene scale } \\
\text { alteration }\end{array}$ & No & $\begin{array}{c}\text { Yes (at } \\
\text { borders) }\end{array}$ & Yes & Yes \\
\hline $\begin{array}{c}\text { View Vol. aspect ratio } \\
\text { alteration }\end{array}$ & Yes & No & No & No \\
\hline
\end{tabular}

4 A novel Approach: Stereo compatible volume CLIPPING

Methods presented in the previous sections are dependent on the displayed content due to the analysis of the scene geometry or the analysis of the disparity of the stereo pair. In real-time rendering, this task has to be automated and executed on each frame, leading to potentially complex and time consuming algorithms.

Definition: In the negative parallax half space, we can define the SCV as the intersection of the left and right view volumes. From a purely geometric point of view, one can solve the D/FO conflict problem optimally by rendering only the SCV.

Approach: Our method does not try to avoid situations where frame cancellation occurs; it simply rejects parts of the viewing volume that are subject to $\mathrm{D} / \mathrm{FO}$ conflict. By rejecting these regions, SCVC is independent of displayed assets and does not rely on content or stereo pair disparity analysis: wide environments are treated in the same manner as small convex objects. The method also ensures the widest volume is displayed on both positive and negative parallaxes while aspect ratio is maintained. No special modification of the scene like scaling or translation is done. The view volume aspect ratio is not altered either.

Implementation: Clipping is a common method used to discard parts of polygons rendered outside of the view volume. Modern 3D APIs let the programmer add arbitrary clipping planes 
in the rendering pipeline. To insure that only stereo compatible volume is rendered, we add the left clipping plane of the right eye camera to the clipping planes list when rendering the right image and vice versa. We can notice that the view volume in positive parallaxes' half space is not altered.

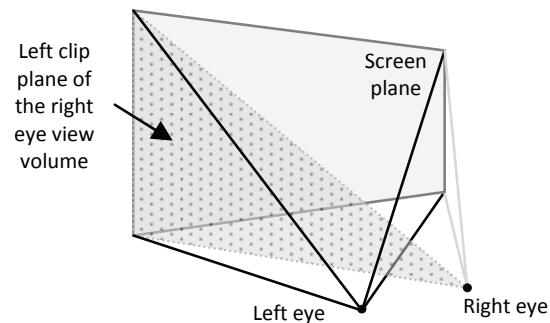

Figure 3. SCVC method: Perspective representation of left eye view volume and left clip plane of right eye view volume. This clip plane is used to clip polygons during the rendering of the left view.

Implementation using the OpenGL fixed pipeline is straightforward in the case of simple stereoscopic rendering (asymmetric frustums and $\mathrm{x}$ axis aligned eyes). The clip plane of interest can be computed using 3 points: center of projection and screen plane top and bottom points (Figure 3.). For non head tracked stereoscopic display, this can be computed once and reused. Cost in terms of computation is marginal for plane equation solving while polygon tests against the additional clip planes stay cost effective when handled by the GPU.

With head tracked displays, the eyes are not necessarily $\mathrm{x}$ axis aligned, making the shape of the intersection of the two view volumes more complex. Adding two clipping planes per view is enough to restrict the rendered space to the SCV. In this case, the eyes' relative position determines the clipping planes that have to be used. We can select at each frame the horizontal clipping plane to use with a simple algorithm (we suppose that left and right eyes are not inverted). For the left view volume rendering: if the right eye is higher to the left eye then the bottom clip plane of the right eye view volume has to be added to the clipping planes list. If the right eye is lower to the left eye, add top clip plane of right eye view volume to the clipping planes list.

\section{Evaluation}

Our objective was to compare SCVC with Black Bands (which seems to be the best state-of-the-art approach, a method recommended in movie industry and already successfully adapted to interactive real-time rendering). Cyclopean scale approaches tend to avoid the problem by altering the scene representation (scaling and translation) so they have not been selected.

Participants: The evaluation was conducted using a panel of 22 participants, 19 males and 3 females aged from 18 to 37, from two groups: naive and initiated users. The 13 naive users had no knowledge of rendering techniques and have very limited experience with stereoscopy. The 9 expert users are university students who have followed real-time rendering courses with explanations of stereoscopic rendering mathematics and algorithms. Subjects suffering from binocular vision pathology were excluded.

Experimental apparatus: The experiment apparatus consists of a CRT screen and Stereographics ${ }^{\mathrm{TM}}$ shutter glasses connected to a computer with an OpenGL stereo enabled graphic board. The user is positioned seated with his head aligned with the screen center at about $80 \mathrm{~cm}$. The test application written with OpenGL fixed pipeline shows a sphere of $6 \mathrm{~cm}$ in diameter to the user, moving in the foreground of a plane. The sphere is positioned at $15 \mathrm{~cm}$ from the screen plane in the negative parallax half view volume and moves slowly on the $\mathrm{x}$ axis with a sine movement from the left to right border. A simple checkerboard texture is used to map the scene objects to let the user fuse left and right images more easily. Care is also taken to set lighting and contrast of the scene at relatively low values to reduce crosstalk (aka ghosting effect) between left and right views. At runtime, the software lets the user toggle the method used by typing a corresponding key. The three methods proposed are Control, SCVC and Black Bands (Figure 4).

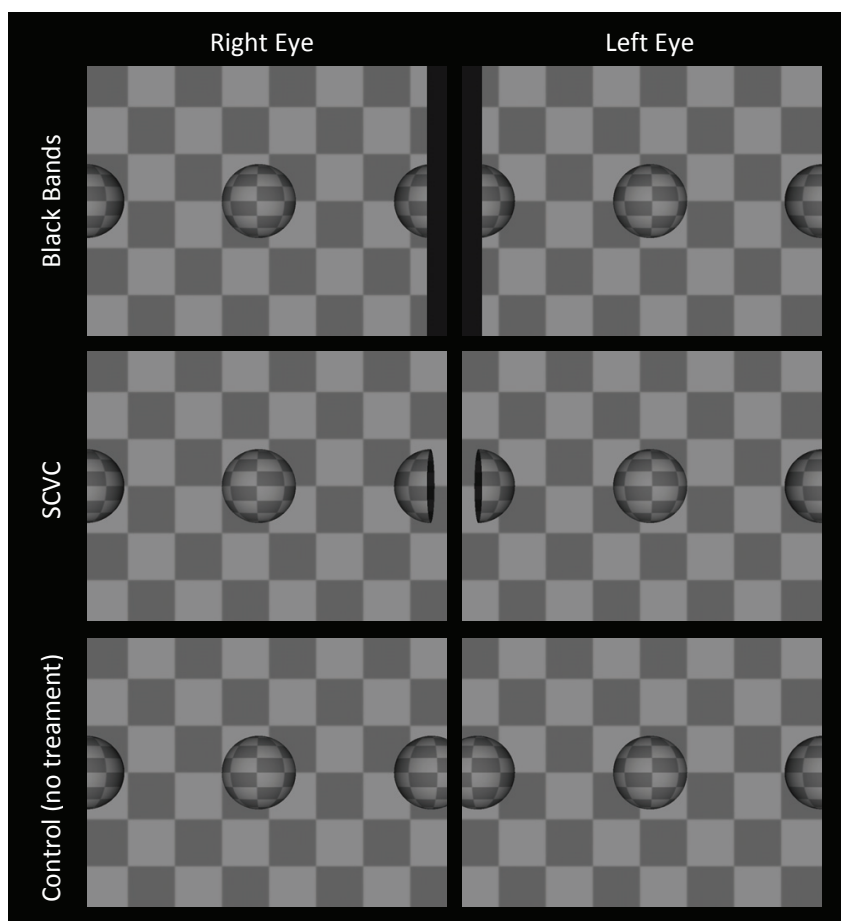

Figure 4. Stereoscopic images in the 3 experimental conditions:

Black Bands (state of the art method), SCVC, and Control (no treatment). A reader comfortable with cross eye stereo can fuse left and right images to see the effects.

Procedure: The experiment starts with a phase where the user can switch between methods as he wishes. Then he is asked open questions about the different methods. Finally he is asked to evaluate different criteria for each method by giving them a score. During the question and evaluation phase, the user remains free to switch from one method to another as he likes.

Data collected: A subjective questionnaire was proposed in which participants had to grade from 1 (low appreciation) to 7 (high appreciation) the three techniques (SCVC, Control and Black Bands) according to 5 subjective criteria: (a) Relief Quality, (b) Relief Quality at Borders, (c) Aesthetic, (d) Eye Strain perceived and (e) Global Appreciation. Figure 5 shows the results concerning the grades (Likert-scale) obtained by the three different techniques for each of the subjective criteria.

Results: A one-way ANOVA was performed on the three different methods (SCVC, Control and Black Bands). A significant effect was found for all criteria, except Eye Strain criterion $(\mathrm{F}(2,21)=2.31$, p-value $=0.109)$. Concerning Relief Quality criterion, a significant effect was found $(\mathrm{F}(2,21)=4.75$, $\mathrm{p}$ value $=0.012$ ). A post-hoc analysis using Tukey's procedure showed that the grading for SCVC method was significantly higher than for Control method ( $\mathrm{p}$-value $=0.02$ ) and Black Bands 
method ( $p$-value $=0.03)$. There was no significant adjusted $p$-value between Control and Black Bands methods.

Concerning Relief Quality at Borders criterion, a significant effect was found $(F(2,21)=9.15$, p-value $=0.0003)$. A post-hoc analysis using Tukey's procedure showed that the grading for SCVC method was significantly higher than for Control method $(p$-value $=0.001)$ and Black Bands method $(p$-value $=0.001)$. There was no significant adjusted p-value between Control and Black Bands methods.

Concerning Aesthetic criterion, a significant effect was found $(\mathrm{F}(2,21)=7.69$, $\mathrm{p}$-value $=0.001)$. A post-hoc analysis using Tukey's procedure showed that the grading for SCVC method was significantly higher than for Control method ( $\mathrm{p}$-value $=0.0006$ ). There were no significant adjusted $\mathrm{p}$-values between Control and Black Bands methods, and SCVC and Control methods.

Concerning Global Appreciation criterion, a significant effect was found $(\mathrm{F}(2,21)=12.018$, p-value $<0.0001)$. A post-hoc analysis using Tukey's procedure showed that the grading for SCVC method was significantly higher than for Control method (p-value $=0.0004)$ and Black Bands method (p-value $=0.0001)$. There was no significant adjusted p-value between Control and Black Bands methods.

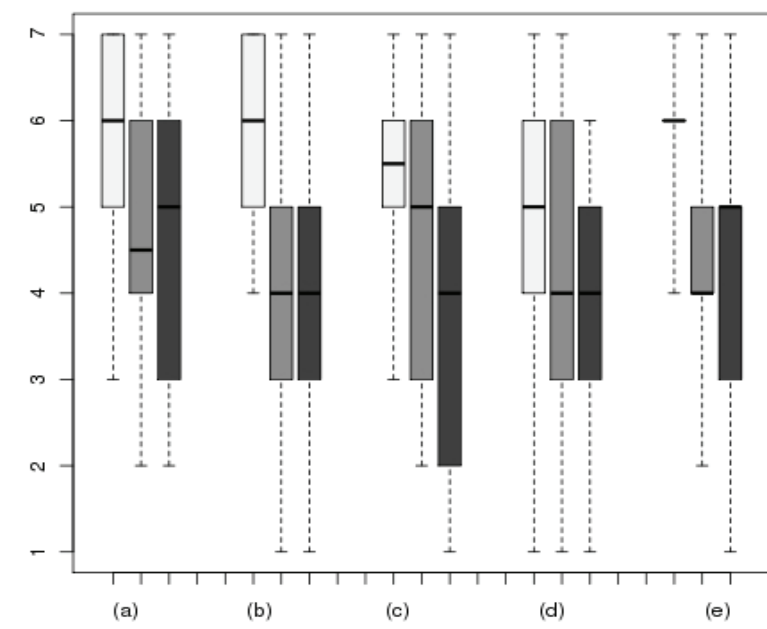

Figure 5. Results for the subjective questionnaire for the three different techniques (1) SCVC (white), (2) Control (light grey) and (3) Black Bands (dark grey), with respect to a Likert-scale grading. The subjective criteria are (a) Relief Quality, (b) Relief Quality at Borders, (c) Aesthetic, (d) Eye Strain perceived and (e) Global Appreciation. Each boxplot is delimited by the quartile ( $25 \%$ and $75 \%$ without outliers) of the distribution of the condition over the individuals. The median is also represented for each technique.

Discussion: The Black Bands method score is surprisingly low. The marks seem to tell us that the method does not solve the problem and that the perception at the screen border is even worse than when no treatment is applied. We think that this could be explained by the use of a CRT screen with white surrounds for the evaluation. When applied to projected images in dark rooms (theaters), the black bands make the user fuse the black band edges with image edges of the same color and luminosity. Besides, users' comments have also highlighted the modification of the view volume by the Blacks Bands, one declaring "I feel like I'm looking at a 9/16 TV'.

The SCVC results confirm that SCVC solves the D/FO conflict problem giving a better depth perception of the sphere in situations when it is partially occluded by the screen borders. Some users have complained of the "slicing" effect induced by the clipping. This can be disturbing depending on the experience the application designers want to give to the user. For applications centered on photorealism or visual attractiveness, the slicing effect might represent a drawback. Some improvements are possible to minimize this problem. For instance, sliced edges could be filled or progressively dissolved. Clipping along a plane orthogonal to the screen is another option, although it would make the method dependent on scene analysis to define the clipping plane. Future work is now necessary to improve this aspect of the method. Nevertheless, applications that do not focus on aesthetic aspects, such as in CAD software, could already benefit from SCVC directly.

\section{Conclusion}

Frame cancellation is a common problem we face in stereoscopic displays. Recent solutions investigated in the movie sector are not easily applicable to real-time rendering and the few methods designed to target VR still suffer from drawbacks and are not always applicable to every environment. We have proposed SCVC, a novel method that solves the problem in a different manner: it only renders the part of the viewing volume that is free of D/FO conflict. This approach does not rely on the analysis of the stereo disparity or the analysis of the scene geometry.

A subjective evaluation conducted with 22 participants has shown scores in favor of SCVC for various criteria. SCVC notably improved global appreciation and user's depth perception near the screen edges.

At a time when stereoscopic capable hardware is available from the mass consumer market, the $\mathrm{D} / \mathrm{FO}$ conflict will become more noticeable. Thanks to its simplicity of implementation, SCVC could target a wide range of rendering software from VR applications to game engines and CAD softwares.

Future work concerns integration of SCVC with realistic rendering. Programmable graphic pipeline could be of help to refine clipped edge rendering though effects like fading or appropriate filling. An advanced evaluation has to be conducted, using a collection of static scenes in conjunction with subjective and quantitative evaluation methods for the eye strain parameter [10]. The influence of surrounds in the Black Bands method is another point to clarify. An experimental campaign confronting different display setups (projector, monitor) and evaluation conditions (dark, bright rooms) should also be conducted.

\section{REFERENCES}

[1] N. A. Valyus, Stereoscopy, The Focal Press, London, 1966.

[2] Z.J. Wartell. Stereoscopic Head-Tracked Displays: Analysis and Development of Display Algorithms, in Technical report from Georgia Institute of Technology, 2002.

[3] L. Lipton, Vertical surround parallax correction. Stereoscopic Displays and Virtual Reality Systems XIV. In Proceedings of the SPIE Conference, 2007.

[4] Autodesk. Maya Software: http://www.autodesk.com

[5] Autodesk. Stereoscopic filmmaking whitepaper, The Business and Technology of Stereoscopic Filmmaking, 2008.

[6] M. Lang, A. Hornung, O. Wang, S. Poulakos, A. Smolic and M. Gross. Nonlinear Disparity Mapping for Stereoscopic 3D, ACM Transactions on Graphics, 2010.

[7] M. Rubinstein, A. Shamir and S. Avidan. Improved Seam Carving for Video Retargeting, ACM Transactions on Graphics, 2008.

[8] J.D. Mulder and Robert Van Liere. Enhancing Fish Tank VR. In Proceedings of the IEEE Virtual Reality Conference. 2000.

[9] C. Ware, D. Fleet. Integrating Flying and Fish Tank Metaphors with Cyclopean Scale, in Proceedings of the Computer Graphics International Conference, 1997.

[10] R.S. Kennedy, N.E. Lane, K.S. Berbaum, and M.G. Lilienthal. Simulator sickness questionnaire: an enhanced method for quantifying simulator sickness. International Journal of Aviation Psychology, 1993. 